\title{
A UNIQUENESS CRITERION FOR ORDINARY DIFFERENTIAL EQUATIONS IN BANACH SPACES
}

\author{
M. ARRATE
}

\begin{abstract}
A uniqueness theorem for the Cauchy problem for ordinary differential equations in complex Banach spaces is given. This paper generalizes and extends a number of known results.
\end{abstract}

1. Introduction. We shall concern ourselves with the initial value problem

$$
x^{\prime}(t)=f(t, x(t)), \quad t \in(0, a), \quad x(0)=x_{0},
$$

where $f$ is a function which maps $(0, a) \times E$ into $E$, a Banach space. If $b \in(0, a]$, a solution of $(1)$ in $[0, b)$ is a function $x:[0, b) \rightarrow E$, continuous in $[0, b)$, differentiable in $(0, b)$ which satisfies (1). This definition may be relaxed assuming that $x$ is a solution in Carathéodory's sense (see Goldstein [6]).

Recently Medeiros [10] and Díaz and Weinacht [4] have studied uniqueness conditions for (1) in a complex Hilbert space. Their results have been extended by Goldstein [6], [7] to real or complex Banach spaces.

On the other hand, Nagumo's classic criterion has been modified by Bownds and Metcalf [3], Rogers [11], Gard [5] and Bernfeld, Driver and Lakshmikantham [2].

The present paper gives a uniqueness theorem which contains those of Goldstein as particular cases and extends to Banach spaces those of Gard and Rogers.

2. Preliminary results. Let $E$ be a complex Banach space, with dual $E^{*}$. We denote by $\langle x, \psi\rangle$ the image of $x \in E$ by $\psi \in E^{*}$. For each $x \in E$ let $J(x)$ denote the (nonempty) set of all $\psi \in E^{*}$ such that

$$
\langle x, \psi\rangle=\|x\|^{2}=\|\psi\|^{2} \text {. }
$$

We call $J$ the duality mapping of $E$. If $f$ is an operator we write its domain as $D(f)$. Let $f: D(f) \subset E \rightarrow E$; we say that $f$ is dissipative if for $x, y$ given in $D(f)$ there exists $\psi \in J(x-y)$ such that

$$
\operatorname{Re}\langle f(x)-f(y), \psi\rangle<0 .
$$

When $E$ is a Hilbert space, $J$ is the identity and then $f$ is dissipative provided

$$
\operatorname{Re}\langle f(x)-f(y), x-y\rangle<0 \quad \forall x, y \in D(f) .
$$

The following result, due to Kato $[9$, p. 510] will be used in the proof of the theorem.

Received by the editors February 20, 1980.

AMS (MOS) subject classifications (1970). Primary 34A10, 34K05.

(C) 1981 American Mathematical Society $0002-9939 / 81 / 0000-0117 / \$ 02.00$ 
Lemma. Assume that $u$ has a weak derivative $u^{\prime}(s) \in E$ at $t=s$, and that $\|u(\cdot)\|$ is differentiable at $t=s$. Then

$$
\|u(s)\|[d\|u(t)\| / d t]_{t=s}=\operatorname{Re}\left\langle u^{\prime}(s), \psi\right\rangle
$$

for each $\psi \in J(u(s))$.

\section{The uniqueness theorem.}

THEOREM. Let $\phi$ be a function continuous in $[0, a)$, differentiable in $(0, a)$, and such that $\phi(0)=0, \phi(t)>0$ for $t>0$. Assume that $u(t), v(t)$ are two solutions of (1) in $[0, b), b \in(0, a)$ verifying

(i) $\|f(t, u(t))-f(t, v(t))\|=o\left[\phi^{\prime}(t)\right]$.

Then, if

(ii) $f(t, \cdot)-\phi^{\prime}(t) I / \phi(t)$ is dissipative for all $t \in(0, b)$ it follows that $u(t) \equiv v(t)$.

Proof. First, note that (ii) is equivalent to the existence, for each pair $x$, $y \in D(f(t, \cdot))$, of a $\psi \in J(x-y)$ such that

$$
\operatorname{Re}\langle f(t, x)-f(t, y), \psi\rangle\left\langle\phi^{\prime}(t)\|x-y\|^{2} / \phi(t) .\right.
$$

Let $u(t), v(t)$ be two solutions of (1). We may assume, without loss of generality, that $u(t) \neq v(t)$ for $t \in(0, b)$. Define

$$
m(t)= \begin{cases}\frac{1}{2}\left[\frac{\|u(t)-v(t)\|}{\phi(t)}\right]^{2} & \text { if } t \in(0, b), \\ 0 & \text { if } t=0 .\end{cases}
$$

For each $\xi \in E^{*}$, L'Hôpital's rule and (i) yield

$$
\begin{aligned}
\lim _{t \rightarrow 0+} \xi\left[\frac{u(t)-v(t)}{\phi(t)}\right] & =\lim _{t \rightarrow 0+} \frac{(\xi[u(t)-v(t)])^{\prime}}{\phi^{\prime}(t)} \\
& =\lim _{t \rightarrow 0+} \xi\left[\frac{f(t, u(t))-f(t, v(t))}{\phi^{\prime}(t)}\right]=0
\end{aligned}
$$

and therefore

$$
\frac{u(t)-v(t)}{\phi(t)} \stackrel{w}{\rightarrow} 0 .
$$

Now, as a consequence of the Banach-Steinhaus theorem [1, p. 255], each weakly convergent sequence is bounded, and hence for any sequence $t_{n} \rightarrow 0+$ there exists $M>0$ such that

$$
\left\|\frac{u\left(t_{n}\right)-v\left(t_{n}\right)}{\phi\left(t_{n}\right)}\right\| \leqslant M, \text { for } n=1,2,3, \ldots
$$

We use Kato's lemma to obtain that for each $t \in(0, b)$ and each $\psi \in$ $J(u(t)-v(t))$

$$
\|u(t)-v(t)\| \frac{d}{d t}\|u(t)-v(t)\|=\operatorname{Re}\left\langle u^{\prime}(t)-v^{\prime}(t), \psi\right\rangle .
$$


Apply again L'Hôpital's rule to get

$$
\begin{aligned}
\lim _{t \rightarrow 0+} m(t) & =\frac{1}{2} \lim _{t \rightarrow 0+} \frac{\|u(t)-v(t)\|^{2}}{[\phi(t)]^{2}} \\
& =\frac{1}{2} \lim _{t \rightarrow 0+} \frac{\|u(t)-v(t)\| \frac{d}{d t}\|u(t)-v(t)\|}{\phi(t) \phi^{\prime}(t)} \\
& =\frac{1}{2} \lim _{t \rightarrow 0+} \operatorname{Re}\left\langle\frac{u^{\prime}(t)-v^{\prime}(t)}{\phi^{\prime}(t)}, \frac{\psi}{\phi(t)}\right\rangle \\
& \leqslant \frac{1}{2} \lim _{t \rightarrow 0+}\left\|\frac{u^{\prime}(t)-v^{\prime}(t)}{\phi^{\prime}(t)}\right\|\left\|\frac{u(t)-v(t)}{\phi(t)}\right\| .
\end{aligned}
$$

In the last term the first factor tends to zero by (i) and upon comparison with (2) we see that $\lim _{t \rightarrow 0+} m(t)=0$ and so $m(t)$ is continuous in $[0, b)$. Hence for $t>0$, the mean value theorem implies there is an $s$ in $(0, t)$ such that

$$
\begin{aligned}
0<m(t)=t m^{\prime}(s)=t \frac{1}{\phi(s)}(\operatorname{Re}\langle f(s, u(s))-f(s, v(s)), \psi\rangle & \\
\left.-\frac{\phi^{\prime}(s)}{\phi(s)}\|u(s)-v(s)\|^{2}\right) & \leqslant 0 .
\end{aligned}
$$

This is a contradiction and therefore $u(t) \equiv v(t)$ in $[0, b)$.

Some well-known theorems of uniqueness can be obtained as corollaries of the preceding theorem. For instance

Corollary 1 (Goldstein [7]). Assume that for some $n \in N, f(t, \cdot)-n I / t$ is dissipative for each $t \in(0, b)$. Then, given $u_{0}, u_{1}, \ldots, u_{n} \in E$ there is at most $a$ solution of $(1)$ in $[0, b)$ such that $u^{(k)}(0)$ exists for $k=0,1, \ldots, n$ and $u^{k}(0)=u_{k}$, $k=0,1, \ldots, n$.

Proof. Take $\phi(t)=t^{n}$. Taylor expansions reveal that

$$
\begin{aligned}
& u^{\prime}(t)=\sum_{k=1}^{n} \frac{t^{k-1} u^{(k)}(0)}{(k-1) !}+o\left(t^{n-1}\right)=\sum_{k=1}^{n} \frac{t^{k-1}}{(k-1) !} u_{k}+o\left(t^{n-1}\right), \\
& v^{\prime}(t)=\sum_{k=1}^{n} \frac{t^{k-1} v^{(k)}(0)}{(k-1) !}+o\left(t^{n-1}\right)=\sum_{k=1}^{n} \frac{t^{k-1}}{(k-1) !} u_{k}+o\left(t^{n-1}\right)
\end{aligned}
$$

where $u, v$ are solutions satisfying the conditions of the corollary. Clearly

$$
\|f(t, u(t))-f(t, v(t))\|=o\left(t^{n-1}\right)
$$

and the theorem applies.

Corollary 2. Let $\phi$ satisfy the conditions of the theorem. Suppose also that

(i) $f(t, x)=h(t)+o\left[\phi^{\prime}(t)\right]$ as $(t, x) \rightarrow\left(0+, x_{0}\right)$,

(ii) $f(t, \cdot)-\phi^{\prime}(t) I / \phi(t)$ is dissipative for each $t \in(0, a)$.

Then (1) has at most one solution. 
This corollary extends a theorem given by Gard [5] for the case $E=R^{n}$. When $\phi(t)=t$, Nagumo's criterion is obtained, and when $\phi(t)=e^{-1 / t}$ we arrive at Roger's result [11].

\section{REFERENCES}

1. G. Bachman and L. Narici, Functional analysis, Academic Press, New York, 1966.

2. S. R. Bernfeld, R. D. Driver and V. Lakshmikantham, Uniqueness for differential equations, Math. Systems Theory 9 (1976), 359-367.

3. J. M. Bownds and F. T. Metcalf, An extension of the Nagumo uniqueness theorem, Proc. Amer. Math. Soc. 27 (1971), 313-316.

4. J. B. Diaz and R. J. Weinacht, On nonlinear differential equations in Hilbert spaces, Applicable Anal. 1 (1971), 31-41.

5. T. Gard, A generalization of the Nagumo uniqueness criterion, Proc. Amer. Math. Soc. 70 (1978), 167-172.

6. J. A. Goldstein, Uniqueness for nonlinear Cauchy problems in Banach spaces, Proc. Amer. Math. Soc. 53 (1975), 91-95.

7. The exact amount of nonuniqueness for singular ordinary differential equations in Banach spaces with an application to the Euler-Poisson-Darboux equations, Nonlinear Equations in Abstract Spaces, V. Lakshmikantham (ed.), Academic Press, New York, 1978, pp. 95-103.

8. V. Lakshmikantham and G. Ladas, Differential equations in abstract spaces, Academic Press, New York, 1972.

9. T. Kato, Nonlinear semigroups and evolution equations, J. Math. Soc. Japan 19 (1967), 508-520.

10. L. A. Medeiros, On nonlinear differential equations in Hilbert spaces, Amer. Math. Monthly 76 (1969), 1024-1027.

11. T. Rogers, On Nagumo's condition, Canad. Math. Bull. 15 (1972), 609-611.

Facultad de Ciencias, Universidad de Valladolid, Valladolid, Spain 